\title{
Asociación de metas y motivaciones académicas en relación con la variable sexo
}

\section{Association of academic goals and motivations in relation to the sex variable}

\section{Carla Patricia Ariza ${ }^{1}$}

\section{Luis Ángel Rueda Toncel ${ }^{2}$}

Jainer Sardoth Blanchar ${ }^{3}$

Universidad de La Guajira, Colombia

Grupo de Investigación GIPRODES

\section{RESUMEN}

Se identifica las asociacion de metas académicas que persiguen los estudiantes de séptimo, octavo, noveno, decimo y undécimo grado, en relación con la variable sexo. La muestra la componen 54 estudiantes (32 hombres, 22 mujeres). Se aplicó el Cuestionario de metas académicas (Hayamizu y Weiner, 1991). Los

1 Doctorante en educación. Magíster en gestión y auditorias ambientales. Carla Patricia Ariza

Correo: cpariza@uniguajira.edu.co ORCID: 0000-00024229-0611 Universidad de La Guajira, Colombia- Grupo de Investigación GIPRODES

2 Doctor en ciencias. Magíster en mercadeo. Luis Ángel Rueda Toncel

Correo: luisangelrueda@uniquajira.edu.co ORCID: 00000002-1686-1943 Universidad de La Guajira, ColombiaGrupo de Investigación GIPRODES

3 er Sardoth Blanchar

Correo: jsardoth@uniguajira.edu.co ORCID: 0000-00033233-8893 Universidad de La Guajira, Colombia- Grupo de Investigación GIPRODES resultados indican que existe una diferencia significativa entre los hombres y mujeres, por lo que las intenciones y metas académicas son diferentes según el sexo.

PALABRAS CLAVE: rendimiento académico, metas de aprendizaje, metas de refuerzo social, metas de logro

\section{ABSTRACT}

The objective is to identify the association of academic goals pursued by students in seventh, eighth, ninth, tenth and eleventh grade, in relation to the variable sex. The sample is made up of 54 students (32 men, 22 women). The Academic Goals Questionnaire was applied (Hayamizu and Weiner, 1991). The results indicate that there is a significant difference between men 
and women, so that academic intentions and goals are different according to gender.

KEY WORDS: academic performance, learning goals, social reinforcement goals, achievement goals

\section{INTRODUCCIÓN}

La motivación es uno de los principales factores que intervienen en el proceso de aprendizaje $y$ el rendimiento académico (Arias A.V., Cabanach, R. G., Lozano, A. B., \& Pérez, J. C. $N, 1997)$. Bandura (1993), desarrollo la teoría cognoscitiva social que considera la motivación como el producto de dos fuerzas principales: la expectativa del individuo para alcanzar una meta y el valor de esta. Una meta académica se puede considerar como un patrón integrado de ideas, atribuciones y sentimientos que determinan las reacciones afectivas, cognitivas y conductuales del sujeto ante sus resultados de éxito o fracaso (García Rodríguez, 1998).

El Cuestionario de Metas Académicas (CMA) es un instrumento elaborado en función de la teoría de Dweck por Hayamizu y Weiner, (1991) bajo el nombre de Questionnaire to Measure achievement Goal Tendencie. Este, luego, fue traducido por Núñez y González-Pienda, (1994) y desde entonces ha sido utilizado en múltiples investigaciones.

Este instrumento se compone de 20 ítems, donde los ocho primeros están centrados en metas intrínsecas y los doce restantes, en metas extrínsecas. Da cuenta de tres tipos de metas formadas por tres factores: Factor I: Metas de aprendizaje (ítems 1 al 8); Factor II: Metas de logro (Ítems 9, 15, 16, 17, 18, 19, 20); Factor III: Metas de refuerzo social (ítems 10 al 14).

Barberá (1998), quien en su libro Psicología del género plantea que la asociación de metas académicas con el sexo, tienen una marcada tendencia en los hombres hacia el reconocimiento público. mientras que las mujeres, valoran mas las cosas bien hechas. Pocos estudios permitan analizar las diferencias entre la asociación de metas académicas y motivación con el sexo o genero de los estudiantes. Bea et al., (2003) desarrollaron una investigación con estudiantes españoles, cuyos resultados demostraron que las mujeres valoraban más las metas educativas e interpersonales, mientras que los hombres presentan mayor interés por las actividades que les otorguen prestigio social.

\section{MATERIAL Y MÉTODOS}

Clasificamos las variables en dos tipos: la variable independiente que viene a ser el sexo y la variable dependiente, que corresponde a las metas académicas del alumnado de secundaria.

\section{Se busca evaluar las siguientes hipótesis}

$\mathrm{H}_{0}$ : No hay diferencia estadísticamente significativa entre las metas académicas del alumnado de secundaria y la variable sexo.

$\mathrm{H}_{1}$ : Hay diferencia estadísticamente significativa entre las metas académicas del alumnado de secundaria y la variable sexo.

Los participantes corresponden a un total de 54 alumnos $(n=54)$ seleccionados mediante muestreo aleatorio en los grados séptimo, octavo, noveno décimo y undécimo grado de la institución educativa María Inmaculada del municipio de Fonseca, con edades comprendidas entre los 12 y los 18 años. El $61,11 \%$ son hombres mientras que el $38,89 \%$ son mujeres.

El instrumento utilizado fue el Cuestionario de Metas Académicas de Hayamizu et al. (1989), adaptado por Hayamizu y Weiner (1991). Este cuestionario contiene tiene 20 ítems para medir tres orientaciones de metas: Metas de Aprendizaje (MA), compuesta por los ítems 1 al 8 los que indican la tendencia de los estudiantes de aprender para adquirir conocimientos, Metas de Logro (ML), compuesta por los ítems 9, 15, 
$16,17,18,19,20$ que reflejan la tendencia de los estudiantes por aprender para obtener buenas notas y, Metas de Refuerzo Social (MRS), que cubre los ítems 10 al 14 y hacen referencia a la tendencia de los estudiantes de aprender para ser aprobados y evitar el rechazo social. Cada ítem del cuestionario se responde en una escala tipo Likert graduada del 1 al 5 , donde 1 significa "nunca" y 5 "siempre".

Tabla 1.

Distribución de los porcentajes en función del sexo, el grado y la edad.

\section{FRECUENCIA $\quad \%$}

SEXO

Hombre 33

Mujer 21

GRADO

Séptimo
Octavo
Noveno
Decimo
Undécimo

EDAD

12
13
14
15
16
17
18

10

9

11

6

10

7

1
61,11

38,89

18,52

20,37

27,78

18,52

14,81

18,52

16,67

20,37

11,11

18,52

12,96

1,85
Se solicitó permiso en la institución educativa María Inmaculada, una institución oficial del nivel secundaria en el municipio de Fonseca (La Guajira), Colombia. Se trabajó con los jóvenes de séptimo, octavo, noveno, decimo y undécimo grado, con edades entre 12 y 18 años. Antes de entregarles el cuestionario se hizo la socialización y explicación de los fines y modo de uso de este, haciendo énfasis en lo importante de no dejar ninguna pregunta sin responder. El cuestionario se aplicó de forma anónima en un tiempo de 10 a 15 minutos, aproximadamente.
Para el manejo estadístico de los datos se realizó el procedimiento Prueba T para muestras independientes, debido a que este nos permite comparar las medias de dos grupos de casos (poblaciones de hombres y mujeres).

Inicialmente se realizó la prueba de hipótesis, donde se definió $\mathrm{H}_{0} \mathrm{y}_{1}$, se determinó el nivel de significancia de $5 \%$. Posteriormente se verificó el supuesto de Normalidad mediante las pruebas de Kolmogorov-Smirnov y Shapiro Wilk y el supuesto de Igualdad de Varianzas (Prueba de Levence), todo esto con el fin de determinar si se 
debía aplicar la prueba t de Student o la prueba de $U$ de Mann-Whitney. Para todos los cálculos estadísticos se empleó el software Statistical Package for the Social Sciences (SPSS) versión 24.0 para Mac.

\section{RESULTADOS Y DISCUSIÓN}

El cuestionario de metas académicas ofrece una fiabilidad (consistencia interna de $\alpha$ de Cronbach) de 0,838 , siendo el mayor valor teórico de Alfa 1 , y en general un valor igual o superior a $\mathbf{0 . 8 0}$ se considera aceptable (Cronbach, 1951).

La distribución en porcentaje de las respuestas de los estudiantes al cuestionario de metas académicas se muestra en tabla 2 , donde podemos ver que, en las Metas de Aprendizaje, el $61,1 \%$ de los estudiantes manifiesta que siempre estudia porque desea saber cosas nuevas, mientras que el 1,9\% nunca estudia porque siente curiosidad. En lo que respecta a las Metas de Refuerzo Social el 71,2 de los estudiantes manifiesta que nunca estudio porque no quiero que el profesor le tenga aversión y el 3,7 siempre estudio porque no quiere que sus amigos se burlen de el. Por ultimo, en la Metas de Logro, en promedio el $72,2 \%$ de los estudiantes siempre estudia porque desea alcanzar una buena posición social en el futuro, y el $16,7 \%$ nunca estudia para ser elogiado por sus padres $y$ profesores.

Los estadísticos del grupo marcan una media de 74,21 para el alumnado hombre y de 62,9 para el alumnado mujeres. El rango de edad de los participantes comprende entre los 12 y los 18 años, con una media de 14,41 y una desviación típica de 1,765 .

Tabla 2

Distribución de porcentajes de las respuestas al cuestionario de Metas académicas

\begin{tabular}{|c|c|c|c|c|c|c|}
\hline & & NUNCA & & & & SIEMPRE \\
\hline \multicolumn{2}{|r|}{ METAS DE APRENDIZAJE } & 1 & 2 & 3 & 4 & 5 \\
\hline & Estudio porque es interesante resolver problemas & & & 37,1 & 44,4 & 18,5 \\
\hline 2. & Estudio porque disfruto descubriendo cuánto he mejorado & 9,3 & 1,9 & 11,1 & 51,9 & 25,9 \\
\hline 3. & Estudio porque deseo saber cosas nuevas & & & 24,1 & 14,8 & 61,1 \\
\hline 4. & Estudio porque me gusta el desafío de los problemas difíciles & & 1,9 & 40,7 & 37 & 20,4 \\
\hline 5. & Estudio porque me siento bien cuando supero obstáculos y fracasos & & & 18,5 & 59,3 & 22,2 \\
\hline 6. & Estudio porque siento curiosidad & 1,9 & 13 & 20,4 & 27,8 & 37 \\
\hline 7. & Estudio porque me gusta emplear mi cabeza & 9,3 & 14,8 & 7,4 & 20,4 & 44,4 \\
\hline $\begin{array}{l}8 . \\
\text { difíc }\end{array}$ & Estudio porque me da alegría cuando puedo resolver un problema & 9,4 & 13,2 & 15,1 & 30,2 & 32,1 \\
\hline
\end{tabular}




\begin{tabular}{|c|c|c|c|c|c|c|c|c|c|}
\hline \multicolumn{10}{|c|}{ METAS DE REFUERZO SOCIAL } \\
\hline \multirow{5}{*}{ 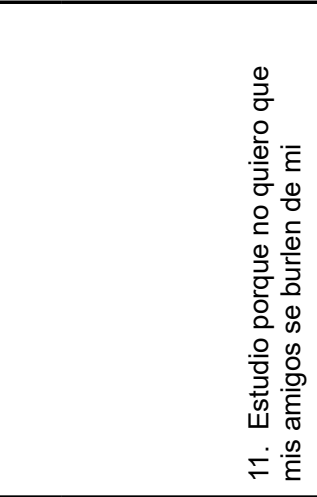 } & \multirow{5}{*}{ 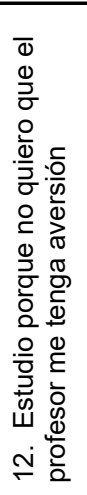 } & \multirow{5}{*}{ 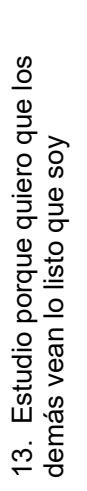 } & \multirow{5}{*}{ 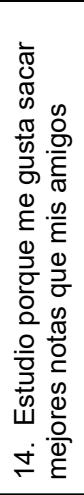 } & $\begin{array}{l}10 . \\
\text { Estudio } \\
\text { porque } \\
\text { quiero } \\
\text { llamar la } \\
\text { atención } \\
\text { de mis } \\
\text { amigos }\end{array}$ & 58,5 & 1,9 & 9,4 & 28,3 & 1,9 \\
\hline & & & & 57,4 & 1,9 & 25,9 & 11,1 & 3,7 & \\
\hline & & & & 71,2 & 1,9 & 21,1 & 5,8 & & \\
\hline & & & & 50 & 24,1 & 7,4 & 7,4 & 11,1 & \\
\hline & & & & 66,7 & 14,8 & 9,3 & 7,4 & 1,9 & \\
\hline \multicolumn{5}{|l|}{ METAS DE LOGRO } & & & & & \\
\hline & & & & $\begin{array}{l}\text { 9. Estudio } \\
\text { porque } \\
\text { quiero ser } \\
\text { elogiado } \\
\text { por mis } \\
\text { padres y } \\
\text { profesores }\end{array}$ & 16,7 & 35,2 & 13 & 14,8 & 20,4 \\
\hline
\end{tabular}

\begin{tabular}{|c|c|c|c|c|c|c|c|c|c|c|}
\hline \multirow{6}{*}{ 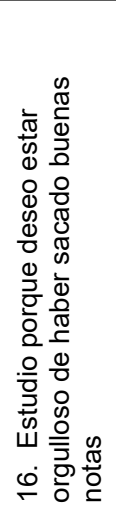 } & \multirow{6}{*}{ 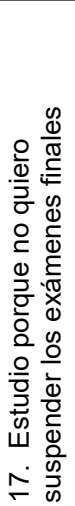 } & \multirow{6}{*}{ 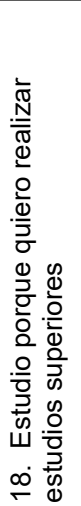 } & \multirow{6}{*}{ 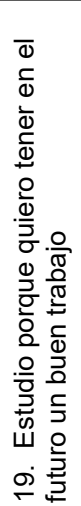 } & \multirow{6}{*}{ 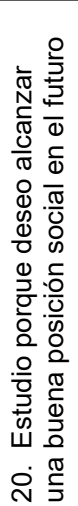 } & $\begin{array}{l}15 . \\
\text { Estudio } \\
\text { porque } \\
\text { quiero } \\
\text { sacar } \\
\text { buenas } \\
\text { notas }\end{array}$ & \multirow[t]{2}{*}{16} & & 4 & \multirow{2}{*}{$\frac{6}{70,4}$} & \multirow[t]{2}{*}{74} \\
\hline & & & & & 14,8 & & 3,7 & 11,1 & & \\
\hline & & & & & 1,9 & 24,1 & 3,7 & 11,1 & 59,3 & \\
\hline & & & & & & & 13 & 20,4 & 66,7 & \\
\hline & & & & & 15,4 & & 9,6 & 1,9 & 73,1 & \\
\hline & & & & & 14,8 & & 9,3 & 3,7 & 72,2 & \\
\hline
\end{tabular}

Tabla 3

Estadísticas del grupo

\begin{tabular}{|c|c|c|c|c|c|}
\hline & Sexo & $\mathrm{N}$ & Media & $\begin{array}{c}\text { Desviación } \\
\text { estándar }\end{array}$ & $\begin{array}{c}\text { Media de error } \\
\text { estándar }\end{array}$ \\
\hline $\begin{array}{c}\text { Metas } \\
\text { Académicas }\end{array}$ & Hombre & 33 & & & 19,132 \\
\hline & Mujer & 21 & 62,9 & 9,3 & 3,331 \\
\hline Edad & & 54 & 14,41 & 1,765 & 2,029 \\
\hline
\end{tabular}


Para corroborar si la variable metas académicas se comporta normalmente en el grupo hombre y en el grupo mujer, se realizó la prueba de normalidad de Kolmogorov-Smirnov y la de Shapiro-Wilk, cuyos resultados se muestran en la tabla 4. Debido a que nuestro grupo de datos es igual mayor a 30 (Serra Añó, Ponce Darós, López Bueno, González Moreno, \&
García Massó, 2014), utilizamos la prueba de Kolmogorov-Smirnov, obteniendo como resultados $\mathrm{P}$-valor menores a 0,05 , lo que nos indica que los datos no provienen de una distribución normal, es decir la variable metas académicas en ambos grupos (hombre y mujer), no se comporta normalmente.

Tabla 4

Prueba de normalidad

\begin{tabular}{|l|l|c|c|c|c|c|c|}
\hline & \multicolumn{1}{|c|}{ Sexo } & \multicolumn{3}{|c|}{ Kolmogorov-Smirnova } & \multicolumn{4}{c|}{ Shapiro-Wilk } \\
\hline & & Estadístico & gl & Sig. & Estadístico & gl & Sig. \\
\hline Metas Académicas & Hombre & 0,171 & 33 & $\mathbf{0 , 0 1 5}$ & 0,809 & 33 & 0 \\
\hline & Mujer & 0,249 & 21 & 0,001 & 0,825 & 21 & 0,002 \\
\hline
\end{tabular}

a Corrección de significación de Lilliefors

Aplicando la prueba de Prueba de Levene de igualdad de varianzas, vemos que el nivel de significación es mayor a 0,05, por lo que asumimos la igualdad de las varianzas. Aun cuando no se cumple el supuesto de normalidad, debido a la robustez de la prueba t de student, podemos aplicarla porque mantiene la validez de los errores de tipo 1 y tipo 2 aunque los valores de la muestra no se distribuyan de forma normal (Serra Añó et al., , 2014).

Tabla 5

Prueba de Levene de igualdad de varianzas

\begin{tabular}{|l|l|c|c|c|c|}
\hline & & $\mathrm{F}$ & Sig. & $\mathrm{t}$ & $\mathrm{gl}$ \\
\hline $\begin{array}{l}\text { Metas } \\
\text { Académicas }\end{array}$ & & & & & \\
\hline & Se asumen varianzas iguales & 2,426 & $\mathbf{0 , 1 2 5}$ & $\mathbf{2 , 5 1 9}$ & 52 \\
\hline & No se asumen varianzas iguales & & & 2,899 & 49,3 \\
\hline
\end{tabular}


Tabla 6

Prueba t para la igualdad de medias

\begin{tabular}{|c|c|c|c|c|c|c|}
\hline & & $\begin{array}{c}\text { Sig. } \\
\text { (bilateral) }\end{array}$ & $\begin{array}{l}\text { Diferencia de } \\
\text { medias }\end{array}$ & $\begin{array}{l}\text { Diferencia de } \\
\text { error estándar }\end{array}$ & \multicolumn{2}{|c|}{$\begin{array}{c}\text { 95\% de intervalo } \\
\text { de confianza de la } \\
\text { diferencia }\end{array}$} \\
\hline & & & & & Inferior & Superior \\
\hline \multirow[t]{2}{*}{$\begin{array}{l}\text { Metas } \\
\text { Académicas }\end{array}$} & $\begin{array}{l}\text { Se asumen } \\
\text { varianzas iguales }\end{array}$ & 0,015 & 11,307 & 4,488 & 2,301 & 20,314 \\
\hline & $\begin{array}{l}\text { No se asumen } \\
\text { varianzas iguales }\end{array}$ & 0,006 & 11,307 & 3,9 & 3,471 & 19,144 \\
\hline
\end{tabular}

La tabla 6 de la igualdad de medias, nos arrojó un nivel de significancia de 0,015. Al ser este valor menor que 0,05 nos indica que se rechaza la $\mathrm{H}_{0}$.

De tal forma que, que hay diferencia estadísticamente significativa entre las metas académicas de los estudiantes (hombres y mujeres) del nivel secundaria, siendo los hombres significativamente mayores $(M=74,21$ $\mathrm{SE}=3,331)$ que las mujeres $(\mathrm{M}=62,9 \mathrm{SE}=$ $2,029 \mathrm{t}(52)=2,519, \mathrm{p}<0,05$.

En varias investigaciones autores como Anderman (1999), Roeser 1996) y Cerezo Rusillo (2004), afirman que los tipos de metas son diferentes entre hombres y mujeres, presentándose en los primeros mayor motivación extrínseca, mientras que las segundas suelen tener mayores niveles de motivación intrínseca.

Caso-Niebla (2007), manifiestan que en la relación entre las metas académicas y la variable sexo, las mujeres presentan mejor rendimiento académico, motivación, y habilidades de estudio que los hombres.
Alemán (2011) por el contrario, concluyen que no existe diferencia alguna en los tipos de orientación de meta en función del sexo. Algunos estudios asocian las metas de aprendizaje al genero femenino, mientras que las metas de rendimiento lo están al género masculino (Wentzel, 1998)

Los resultados de nuestra investigación coinciden con los propuestos por Anderman (1999), Roeser 1996) y Cerezo Rusillo (2004), quienes afirman que los hombres presentan mayor motivación que las mujeres.

\section{CONCLUSIONES}

El objetivo de esta investigación fue conocer las orientaciones de meta académicas de los estudiantes de séptimo, octavo, noveno, decimo y undécimo grado y su relación con el sexo, de forma tal, que se pudo establecer que en su mayoría los, principalmente los hombres, estudian por alcanzar metas de aprendizaje, mas que por lograr un reconocimiento social ante profesores, compañeros y padres. 
Concluyendo que, si existe una diferencia significativa entre las orientaciones de metas académicas de estudiantes hombres y estudiantes mujeres, por lo que las intenciones y metas son diferentes según el sexo.

\section{REFERENCIAS BIBLIOGRÁFICAS}

Alemán, M. J. (2011). Orientaciones motivacionales, rendimiento académico y género en estudiantes de bachillerato. Ciencias Psicológicas , 5 (2), 159-166.

Anderman, L. H. (1999). Social predictors of changes in students' achievement goal orientations. Contemporary Educational Psychology , 24 (1), 21-37.

Arias A.V., Cabanach, R. G., Lozano, A. B., \& Pérez, J. C. N. (1997). Motivación, cognición y aprendizaje autorregulado. Revista Española de Pedagogía, 137-164.

Barberá, E. (1998). Psicología del género. (I. M. Benlloch, Ed.) Barcelona: Ariel.

Bea, M. V. L., de Acedo Lizarraga, M. L. S., \& Martínez, M. D. U. (2003). Desarrollo y validación de un cuestionario de metas para adolescentes. Psicothema, 15(3), 493-499.

Caso-Niebla, J. \&. (2007). Variables que inciden en el rendimiento académico de adolescentes mexicanos. Revista latinoamericana de psicología, , 39 (3), 487-501.

Cerezo Rusillo, M. \&. (2004). Diferencias de género en la motivación académica de los alumnos de Educación Secundaria Obligatoria. Electronic journal of research in educational psychology , 2 (1).

Cronbach, L. J. (1951). Coefficient alpha and the internal structure of tests. psychometrika , 16 (3), 297-334.
García Rodríguez, M.S.-P. (1998). El cuestionario de metas académicas (CMA): un instrumento para la evaluación de la orientación motivacional de los alumnos de Educación Secundaria. Aula Abierta , 71 .

Hayamizu, T. \&. (1991). A test of Dweck's model of achievement goals as related to perceptions of ability. The Journal of Experimental Education , 59 (3), 226234.

Núñez, J. C.-P. (1994). Determinantes del rendimiento académico. Oviedo: SPU.

Roeser, R. W. (1996). Perceptions of the school psychological environment and early adolescents' psychological and behavioral functioning in school: The mediating role of goals and belonging. Journal of educational psychology , 88 (3), 408.

Serra Añó, P., Ponce Darós, M. J., López Bueno, L., González Moreno, L. M., \& García Massó, X. (2014). Prueba T de Student para Muestras Independientes.

Wentzel, K. R. (1998). Social relationships and motivation in middle school: The role of par-ents, teachers, and peers. Journal of educational psychology , 90 (2), 202. 